TITLE:

\title{
The Outcome of Cochlear Implantation for Mitochondrial Disease Patients With Syndromic Hearing Loss.
}

\section{AUTHOR(S):}

Yamamoto, Norio; Okuyama, Hideaki; Hiraumi, Harukazu; Sakamoto, Tatsunori; Matsuura, Hitomi; Ito, Juichi

\section{CITATION:}

Yamamoto, Norio ... [et al]. The Outcome of Cochlear Implantation for Mitochondrial Disease Patients With Syndromic Hearing Loss.. Otology \& neurotology 2015, 36(8): e129e133

\section{ISSUE DATE:}

2015-09

URL:

http://hdl.handle.net/2433/202108

\section{RIGHT:}

This is a non-final version of an article published in final form in [Yamamoto Norio, Okuyama Hideaki, Hiraumi Harukazu, Sakamoto Tatsunori, Matsuura Hitomi, Ito Juichi. The Outcome of Cochlear Implantation for Mitochondrial Disease Patients With Syndromic Hearing Loss. Otology \& Neurotology, 36(8), September 2015, p e129-e133]; The fulltext file will be made open to the public on 1 October 2016 in accordance with publisher's 'Terms and Conditions for Self-Archiving'.; この論文は出版社版でありません。引用の際には出版社版をご確認ご利用ください。; This is not the published version. Please cite only the published version. 


\section{$1 \quad$ Abstract}

2 Objective: To evaluate the outcome and to confirm the validity of cochlear implantation

3 for syndromic deafness in patients with mitochondrial disease.

4 Study design: Retrospective case review

$5 \quad$ Setting: Tertiary referral center

6 Patients: We reviewed medical charts of 367 cochlear implantation cases at Kyoto

7 University Hospital between 1987 and 2012. We identified 5 patients with syndromic

8 mitochondrial disease who underwent cochlear implantation surgery. The mean age of

9 the patients (4 women and 1 man) when they underwent surgeries was 44.4 years

10 (range 30-64years, median 41 years).

11 Interventions: Therapeutic and rehabilitative

12 Main outcome measure: In 4 out of 5 patients, speech perception performance was

13 measured using Japanese vowels, consonant-vowel syllables, and short sentences.

14 Results: Only 1.4\% (5/367) of cochlear implantation cases at Kyoto University Hospital

15 underwent cochlear implantation surgery due to syndromic mitochondrial diseases.

16 Four of those patients showed significantly improved speech perception outcomes, and

17 the beneficial effects of the intervention continued long after surgery. One patient could not perform speech perception test presumably due to poor cognitive function. 
19 Conclusions: Mitochondrial disease patients who underwent cochlear implantation

20 surgery sustained gains in hearing performance even long after surgery. A single

21 patient showed poor postoperative speech perception associated with cognitive problems.

22 Cochlear implantation for mitochondrial disease patients appears to be a viable

23 treatment option in the absence of significant cognitive impairment.

\section{INTRODUCTION}

26 Mitochondrial diseases are caused by mutation of either mitochondrial DNA or of

27 nuclear DNA that encodes genes related to mitochondrial function. Mitochondrial

28 diseases result in dysfunction of the respiratory chains that are important for producing

29 adenocine triphosphate (ATP) in eukaryotic cells (1). More than half of the known mitochondrial diseases cause various levels of sensorineural hearing loss (SNHL) (2) that is classified into either non-syndromic or syndromic hearing loss. Mitochondrial diseases with syndromic hearing loss include mitochondrial encephalomyopathy, lactic acidosis, and stroke-like episodes (MELAS), myoclonic epilepsy with ragged-red fibers (MERRF), maternally inherited diabetes with deafness (MIDD), Kearns-Sayre syndrome (KSS), and chronic progressive external ophthalmoplegia (CPEO). Some mitochondrial diseases can cause severe to profound SNHL, necessitating the use of 
37 cochlear implants for syndromic (3-15), as well as non-syndromic (16-18), mitochondrial deafness.

Most previous case reports suggested that cochlear implantation (CI) has favorable effects in both types of mitochondrial diseases, based solely on the outcome at 1 time point or within at most 2 years after surgery. Although these results are supported by the fact that the SNHL in mitochondrial diseases is attributed to cochlear dysfunction $(19,20)$, mitochondrial diseases with syndromic deafness can affect the central nervous system, including the central auditory pathway, or cause psychomotor regression after CI (1). Thus, the beneficial effects of cochlear implants may further change even after determination of the initial treatment outcome or within several years after surgery, since continued normal function of the central auditory pathway and stable psychological conditions are necessary for successful CI. In this study, we present the outcomes of CI on a longitudinal basis at several time points in cases of syndromic mitochondrial deafness that presented at Kyoto University Hospital and discuss the validity to perform CI for syndromic mitochondrial deafness. 
56 This study was approved by Kyoto University Graduate School and Faculty of Medicine,

57 Ethics Committee (E2359). Medical records of 367 patients who underwent CI at Kyoto

58 University Hospital between 1987 and 2012 were reviewed. Among these, 5 patients

59 were diagnosed with MELAS (3 patients), MIDD (1 patient), and unclassified (1 patient)

60 mitochondrial disease in the Department of Neurology at Kyoto University Hospital.

61

62

Data collection

63 Medical records of 5 patients who underwent CI surgery were reviewed and the

64 following information was extracted: age, sex, perioperative complications, and postoperative speech perception performance at several time points.

66

67 Diagnosis of mitochondrial diseases

68 Mitochondrial diseases were diagnosed by neurologists at Kyoto University Hospital

69 based on genetic tests, muscle biopsies, MRI imaging of the brain, and clinical symptoms—seizures, stroke-like symptoms, recurrent headache, dementia, ataxia, muscle weakness, hemianopsia, diabetes mellitus, conduction disorders of the heart, etc. 
74 Postoperative speech perception performance test

75 Vowels, consonant-vowel (CV) syllables, and short sentences were phonated by a male

76 professional announcer and digitized at a sampling rate of $44.1 \mathrm{kHz}$. These speech

77 samples were presented via speakers at 70 dB SPL using a PowerMac PM-7300/166

computer (Apple Inc., Cupertino, California, USA) in random order; the percentage of correct answers was recorded. In the vowel perception test, 5 Japanese vowels were presented to patients. In the CV syllable perception test, $13 \mathrm{CV}$ syllables-composed of 13 Japanese consonants and the vowel/a/- were presented to patients. In the phrase perception test, 10 short Japanese sentences were arranged to contain 40 different phrases. The vowel and CV syllable perception test used closed sets and the phrase perception test used an open set. These tests were administered at least 6 months after implantation. Unpaired $t$-tests were performed for the statistical analysis and $p$-values below 0.05 were considered statistically significant.

\section{RESULTS}

$90 \quad$ Patient characteristics (Table) 
91 Only $1.4 \%$ (5/367) of CI cases at Kyoto University Hospital underwent CI due to

92 mitochondrial diseases. The patients-1 male and 4 females- ranged from 30 to 64

93 years in age. Four of the patients had m.3243A>G mutation. Three patients were

94 diagnosed with MELAS and one patient was diagnosed with MIDD. One patient (case 4)

95 was not diagnosed with a specific mitochondrial disease because he presented with an

96 atypical set of symptoms (deafness, ataxia, mild cognitive deficits, and paroxysmal

97 supraventricular tachycardia). However, he was diagnosed with a general

98 mitochondrial disease because he showed the m.3243A $>\mathrm{G}$ mutation and ragged-red

99 fibers were observed in his muscle biopsy specimens. Another patient (case 3) refused to

100 undergo a genetic test and a muscle biopsy test. However, she was clinically diagnosed

101 with MELAS due to typical symptoms (stroke-like episodes, seizures, hemiplegia,

102 cognitive deficits, ataxia, short stature, and deafness) and typical MRI imaging findings

103 (basal ganglia calcification, cerebellar atrophy, and chronic infarcts involving multiple

104 vascular territories). Patients in cases 1,2 , and 3 died 12, 4, and 2 years after CI surgery,

105 respectively.

107 Surgical findings

108 We did not find any inner ear anomalies and smooth and complete electrode insertion 
109 was achieved in all patients. Electrically evoked compound action potential (ECAP) was

110 detected in 3 cases. The implants used in the other 2 cases (cases 1 and 3) were

111 incompatible with ECAP measurements.

112 Although susceptibility to malignant hyperthermia in patients with mitochondrial

113 diseases has been reported (21), none of the patients in our series showed malignant

114 hyperthermia. While most inhalation anesthetics and propofol can suppress complexes I

115 and II of mitochondrial respiratory chains (22), none of our patients suffered from any

116 problems.

118 Postoperative speech perception performance test

119 Four out of the five patients showed good performance in the speech perception performance test after CI. The average performance of these 4 patients $(92.5 \%$ for vowels, $45.0 \%$ for CV syllables, and $78 \%$ for sentences, Figure 1) was comparable to that of the other CI patients with post-lingual deafness at Kyoto University Hospital (23) speech perception results were not significantly different between those of a 
127 persisted for at least 8 and 3 years after surgery in case 1 and case 2 , respectively

128 (Figures 2 and 3). Case 4 and case 5, in which the tests were conducted only once, also

129 showed good results in the speech perception performance test 2 years and 1.5 years

130 after surgery, respectively (Figure 4). In case 3 the patient could not participate in the

131 speech perception performance test even 2 years after CI surgery presumably due to the

132 poor cognitive function. This patient's average threshold in the sound field pure-tone

133 audiometry was $30-45 \mathrm{~dB}$ hearing level from $250-4000 \mathrm{~Hz}$ at 1 year after CI surgery.

135 Discussion

136 Possible causes of SNHL by mitochondrial diseases with syndromic hearing loss are

137 cochlear dysfunction and retrocochlear impairment. Several studies suggested that

138 cochlear dysfunction was the more probable cause of SNHL in mitochondrial diseases

139 with syndromic hearing loss $(19,20)$. However, other reports suggested the involvement

140 of retrocochlear impairment based on the increased latency in auditory brain stem

141 response (ABR) (24,25). Favorable outcomes of CI (3-15), including the preserved

142 retrocochlear function shown by an electrically induced middle latency response (MLR)

$143(5,10,12)$, supported the cochlear origin of SNHL in mitochondrial diseases theory. Our 
145 previous reports evaluated outcomes only once within 2 years after surgeries.

146 Since impairment of retrocochlear function may occur many years after CI surgeries

147 and may cause deterioration of CI outcomes, repeated evaluations over a longer period

148 are imperative. In this study, we performed the postoperative speech perception

149 performance test on 2 patients (cases 1 and 2) several times over 8 and 3 years,

150 respectively. These results showed the preservation of retrocochlear function in both

151 MELAS and MIDD patients over extended periods after their CI surgeries. While 5

152 years of follow-up of the CI outcome has been reported for MIDD patients (8), the

153 audiological evaluation in our study showed that even in MELAS, which is considered

154 more severe mitochondrial disease than MIDD (12), the retrocochlear function was

155 preserved over an extended period of time.

156 In addition to the dysfunction of central auditory pathways, cognitive problems should

157 be considered when deciding the indications for CI especially in severe mitochondrial

158 diseases such as MELAS. The cognitive deficit sometimes causes the limited usage of a

159 cochlear implant. The patient in case 3 had a strong desire to recover her hearing ability

160 prior to her CI surgery. However, she could not recognize the importance of using her

161 implant for the establishment of speech perception; she used her implant only several 
1632 year after CI surgery. This was despite her sound field pure-tone audiometry result

164 being comparable to that for the other CI users.

165 Among the 5 patients, 3 died 12, 4, and 2 years after their CI surgeries. The poor

166 prognosis of MELAS (26) raises the problem of cost-effectiveness of CI for syndromic

167 deafness due to mitochondrial diseases. Nevertheless, the long-term preferable

168 outcomes of CI in mitochondrial diseases shown in this study, and the possibility of

169 other severe symptoms caused by mitochondrial disease such as visual disturbance,

170 support the validity of CI for mitochondrial disease patients with hearing loss.

171

172

CONCLUSION

173

174 Mitochondrial disease patients who underwent cochlear implantation surgery sustained

175 gains in hearing performance even long after CI surgery. A single patient had poor

postoperative speech perception associated with cognitive problems. Cochlear

in the absence of significant cognitive function. 


\section{REFERENCES}

181 1. DiMauro S, Schon EA. Mitochondrial disorders in the nervous system. Annual review of neuroscience 2008;31:91-123.

183 2. Chinnery PF, Elliott C, Green GRet al. The spectrum of hearing loss due to mitochondrial DNA defects. Brain 2000;123 ( Pt 1):82-92.

185 3. Yamaguchi T, Himi T, Harabuchi Yet al. Cochlear implantation in a patient with mitochondrial disease--Kearns-Sayre syndrome: a case report. Adv Otorhinolaryngol 1997;52:321-3.

4. Cullington HE. Cochlear implantation of a deaf blind patient with mitochondrial cytopathy. J Laryngol Otol 1999;113:353-4.

5. Rosenthal EL, Kileny PR, Boerst Aet al. Successful cochlear implantation in a patient with MELAS syndrome. Am J Otol 1999;20:187-90; discussion 90-1.

6. Counter PR, Hilton MP, Webster Det al. Cochlear implantation of a patient with a previously undescribed mitochondrial DNA defect. J Laryngol Otol 2001;115:730-2.

7. Hill D, Wintersgill S, Stott Let al. Cochlear implantation in a profoundly deaf patient with MELAS syndrome. J Neurol Neurosurg Psychiatry 2001;71:281.

8. Raut V, Sinnathuray AR, Toner JG. Cochlear implantation in maternal inherited diabetes and deafness syndrome. J Laryngol Otol 2002;116:373-5.

9. Sinnathuray AR, Raut V, Awa Aet al. A review of cochlear implantation in mitochondrial sensorineural hearing loss. Otol Neurotol 2003;24:418-26.

10. Yasumura S, Aso S, Fujisaka Met al. Cochlear implantation in a patient with mitochondrial encephalopathy, lactic acidosis and stroke-like episodes syndrome. Acta Otolaryngol 2003;123:55-8.

11. Karkos PD, Anari S, Johnson IJ. Cochlear implantation in patients with MELAS syndrome. Eur Arch Otorhinolaryngol 2005;262:322-4.

12. Pijl S, Westerberg BD. Cochlear implantation results in patients with Kearns-Sayre

13. Li JN, Han DY, Ji Fet al. Successful cochlear implantation in a patient with MNGIE syndrome. Acta Otolaryngol 2011;131:1012-6.

15. Nishizaki K, Fukushiama K, Oda Yet al. Cochlear implantation for symptomatic hereditary deafness. Acta oto-laryngologica. Supplementum 1999;540:34-7.

214 16. Mancuso M, Filosto M, Forli Fet al. A non-syndromic hearing loss caused by very low 
levels of the mtDNA A3243G mutation. Acta Neurol Scand 2004;110:72-4.

17. Tono T, Ushisako Y, Kiyomizu Ket al. Cochlear implantation in a patient with profound hearing loss with the A1555G mitochondrial mutation. Am $J$ Otol 1998;19:754-7.

18. Ulubil SA, Furze AD, Angeli SI. Cochlear implantation in a patient with profound hearing loss with the A1555G mitochondrial DNA mutation and no history of aminoglycoside exposure. J Laryngol Otol 2006;120:230-2.

19. Sue CM, Lipsett LJ, Crimmins DSet al. Cochlear origin of hearing loss in MELAS syndrome. Ann Neurol 1998;43:350-9.

20. Yamasoba T, Oka Y, Tsukuda Ket al. Auditory findings in patients with maternally inherited diabetes and deafness harboring a point mutation in the mitochondrial transfer RNA(Leu) (UUR) gene. Laryngoscope 1996;106:49-53.

21. Fricker RM, Raffelsberger T, Rauch-Shorny Set al. Positive malignant hyperthermia susceptibility in vitro test in a patient with mitochondrial myopathy and myoadenylate deaminase deficiency. Anesthesiology 2002;97:1635-7.

22. Lerman J. Perioperative management of the paediatric patient with coexisting neuromuscular disease. British journal of anaesthesia 2011;107 Suppl 1:i79-89.

23. Hiraumi H, Tsuji J, Kanemaru Set al. Cochlear implants in post-lingually deafened patients. Acta oto-laryngologica. Supplementum 2007:17-21.

24. Elverland HH, Torbergsen T. Audiologic findings in a family with mitochondrial disorder. Am J Otol 1991;12:459-65.

25. Zwirner P, Wilichowski E. Progressive sensorineural hearing loss in children with mitochondrial encephalomyopathies. Laryngoscope 2001;111:515-21.

26. Yatsuga S, Povalko N, Nishioka Jet al. MELAS: a nationwide prospective cohort study of 96 patients in Japan. Biochimica et biophysica acta 2012;1820:619-24. 


\section{$243 \quad$ FIGURE Legends}

244 Figure 1. Comparison of post-operative speech perception performance test results.

245 The average scores in the post-operative speech perception performance test for vowels,

246 consonant-vowel (CV) syllables, and short sentences were compared between 4

247 mitochondrial disease patients (white boxes, case 1, 2, 4, and 5) and other post-lingually

248 deafened patients (black boxes) who underwent cochlear implantation. Error bars

249 indicate standard deviation. No significant difference in any test was detected between

250 mitochondrial disease patients and other post-lingually deafened patients. Error bars

251 indicate standard deviation.

252

253 Figure 2. Time course of post-operative speech perception performance in case 1.

254 The performances for vowels and sentences were well maintained even 8 years after surgery.

257 Figure 3. Time course of post-operative speech perception performance in case 2.

258 The performances were well maintained 3 years after surgery.

Figure 4. The outcomes of post-operative speech perception performance test for cases 4 
261 and 5.

262 The tests were performed 2 years and 1.5 years after surgery, respectively.

263 


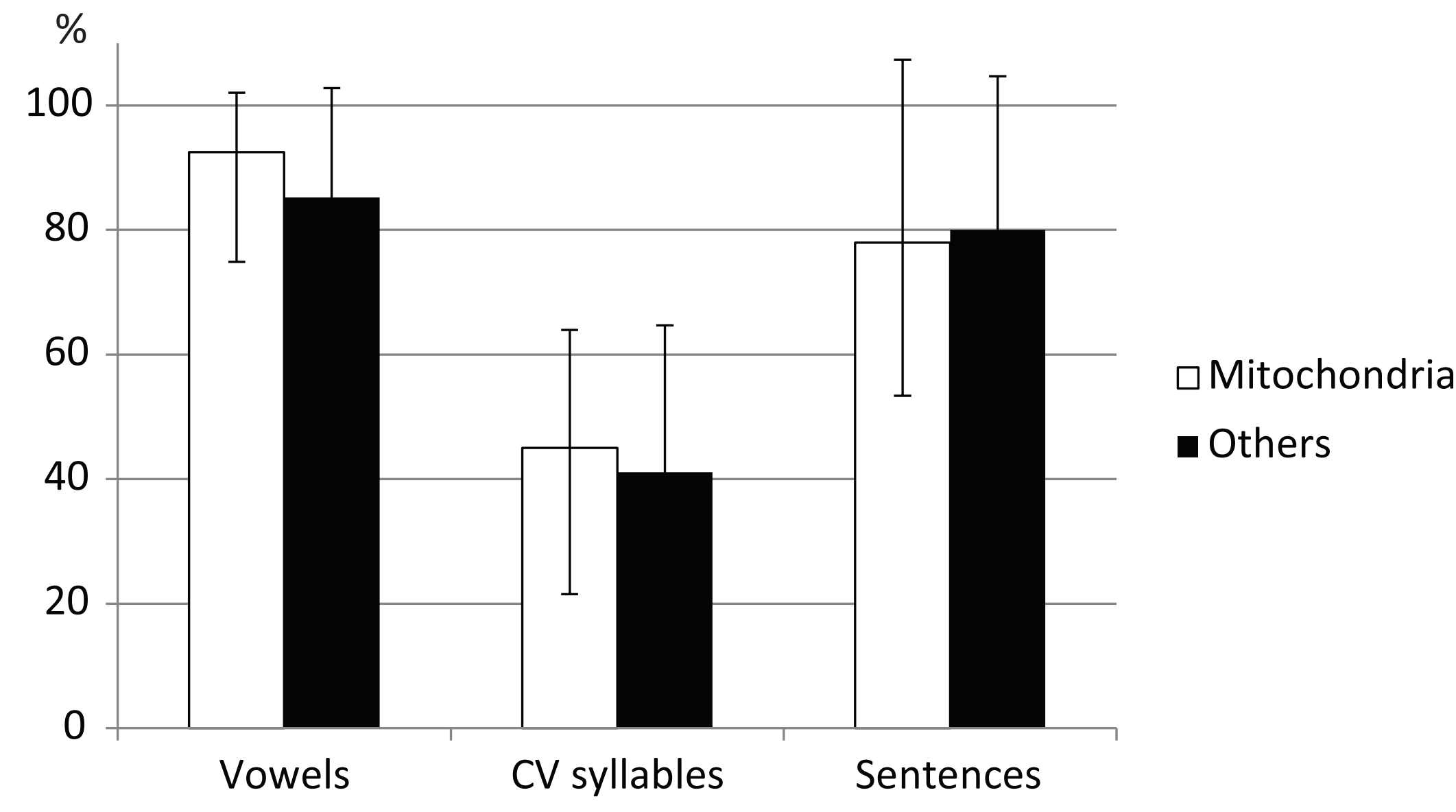




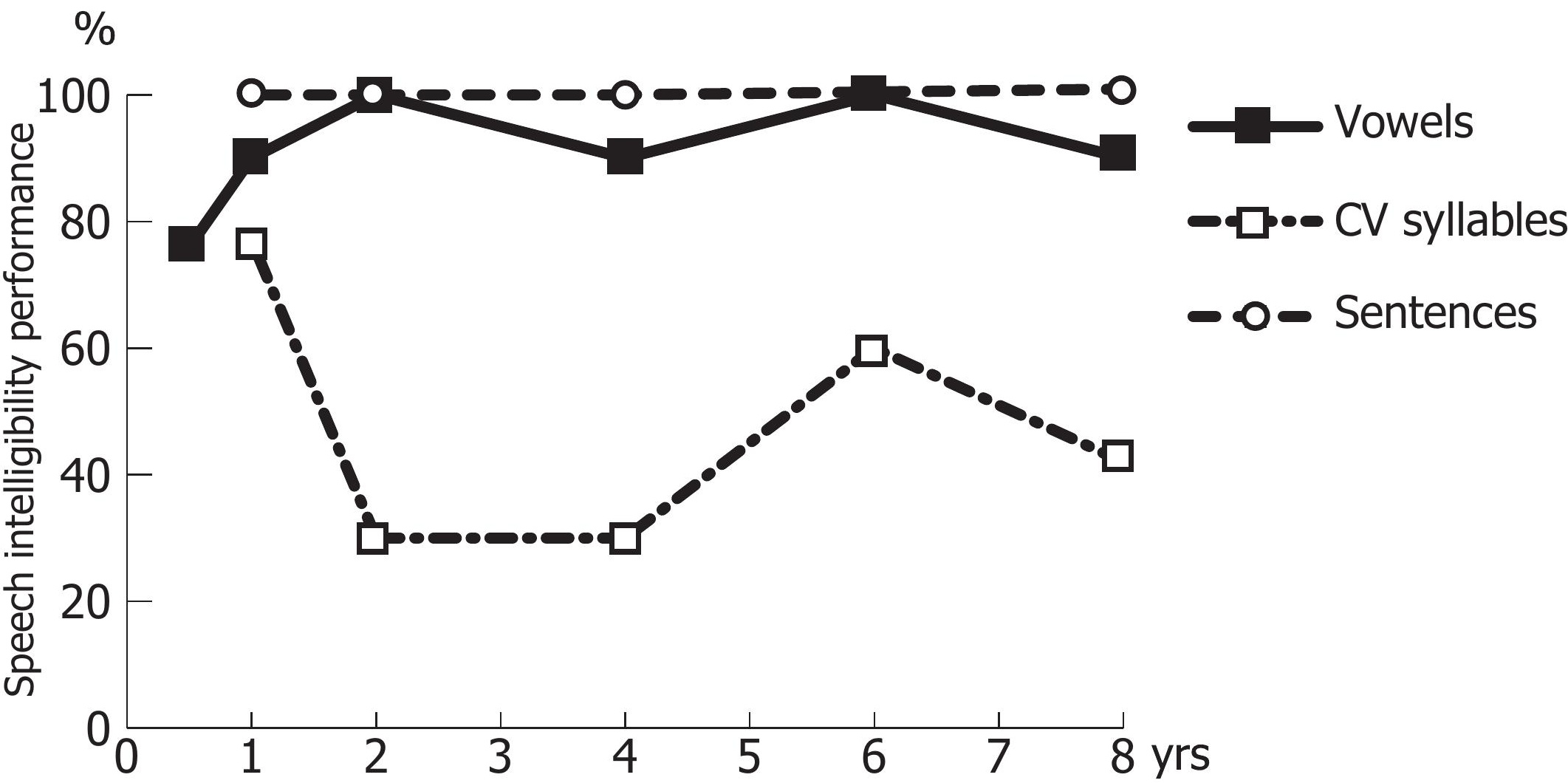




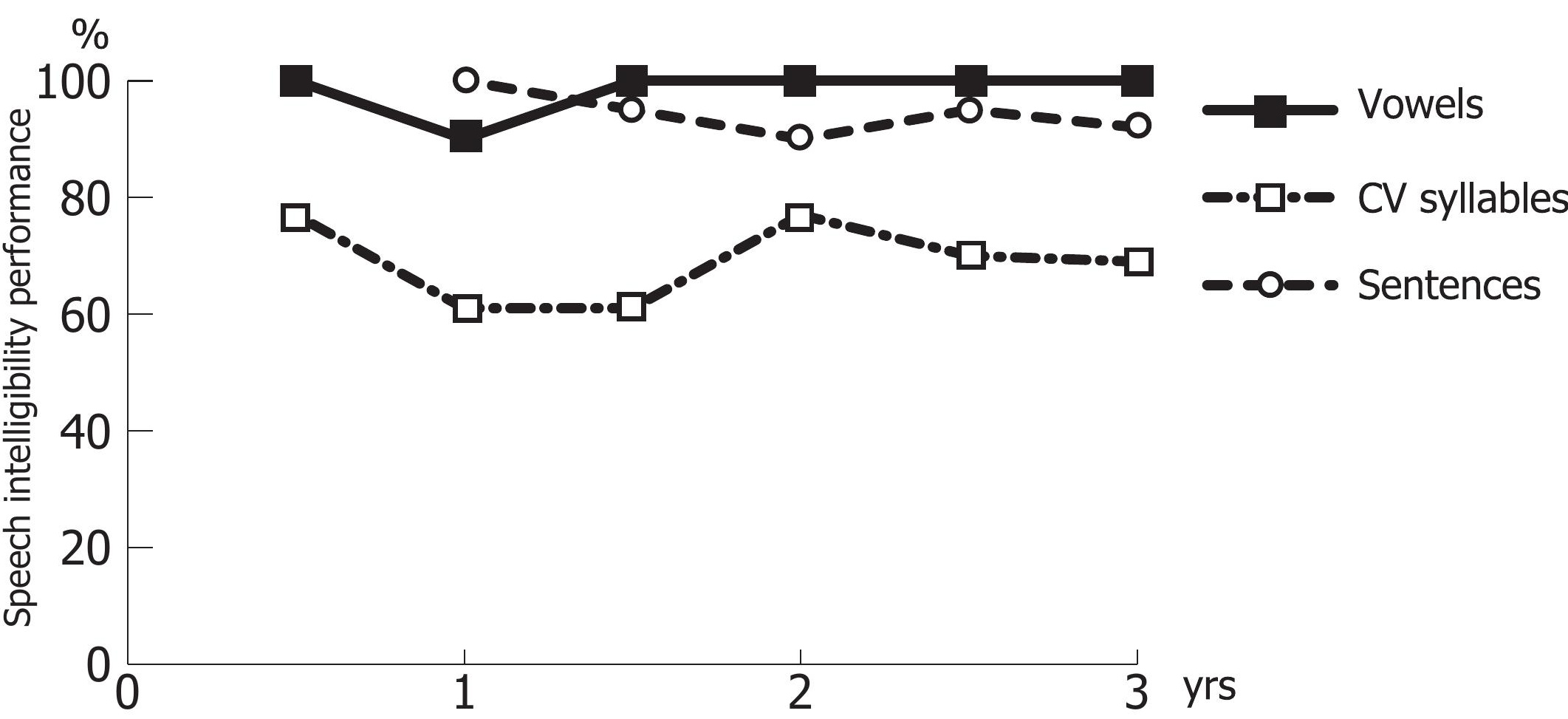




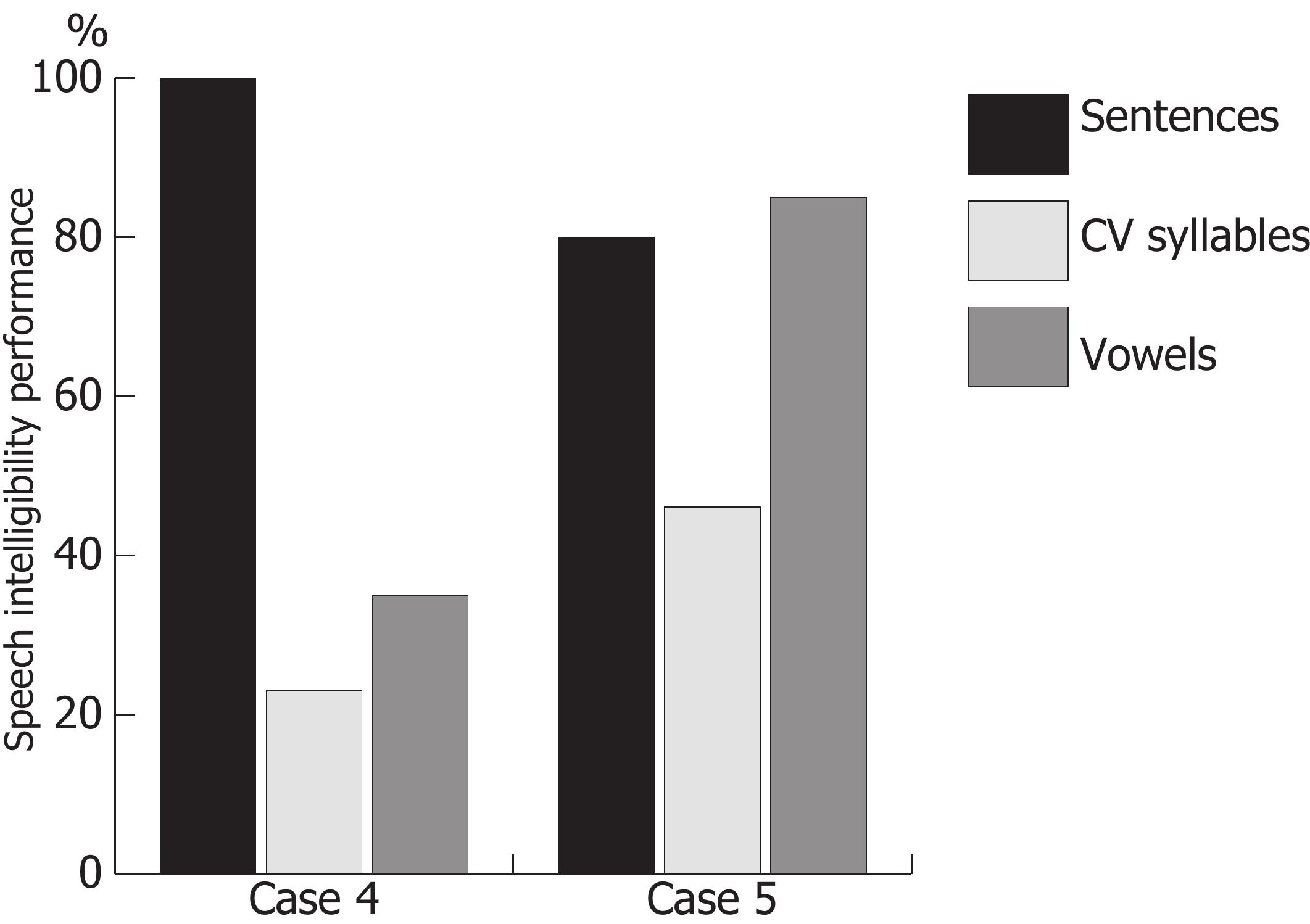


Table

Summary of patients

\begin{tabular}{cccccccc}
\hline Case & Sex & Age & Disease & Mutation & $\begin{array}{c}\text { Duration of } \\
\text { deafness }\end{array}$ & PR & CI \\
\hline $\mathbf{1}$ & F & 41 & MELAS & m.3243A $>$ G & 6 years & - & CI22 \\
$\mathbf{2}$ & F & 64 & MIDD & m.3243A $>$ G & 1 years & - & CI24R \\
$\mathbf{3}$ & F & 41 & MELAS & N.D. & 2 years & +++ & Combi40+ \\
$\mathbf{4}$ & M & 30 & Unclassified & m.3243A $>$ G & 20 years & + & Pulsar100 \\
5 & F & 36 & MELAS & m.3243A $>$ G & 2 years & - & CI24RE(CA) \\
\hline
\end{tabular}

N.D.: not determined; PR: psychomotor regression 